\section{P-24 A CLINICAL AUDIT OF XEROSTOMIA ASSESSMENT AND TREATMENT PRACTICES AMONGST ADVANCED CANCER PATIENTS IN A PALLIATIVE CARE SETTING}

${ }^{1,2}$ Ciarán Kenny, ${ }^{3}$ Narayani Mukerii, ${ }^{1,2}$ Declan Walsh. ${ }^{1}$ Academic Department of Palliative Medicine, Our Lady's Hospice and Care Services, ${ }^{2}$ School of Medicine, Trinity College Dublin; ${ }^{3}$ School of Medicine and Medical Science, University College Dublin

10.1136/bmjspcare-2017-00133.24

Background Xerostomia is the subjective sensation of dry mouth. It is the fourth most common symptom in advanced cancer patients and impacts negatively on physical and psychosocial wellbeing. Older age and polypharmacy are risk factors for dry mouth and are common in advanced disease. This study aims to evaluate prevalence of xerostomia, as well as compliance with assessment and treatment practices.

Methods A retrospective chart audit was conducted on 173 admissions from an in-patient palliative care unit. Data were collected pertaining to patient demographics, cancer diagnosis, medications, oral health assessment and xerostomia treatment. Audit standards were based on local policy as follows: Oral Health Assessment Tool (OHAT) completed on all patients; OHAT completed within one day of admission; oral care plan completed if problem diagnosed; xerostomia treatment prescribed where necessary. Descriptive statistics were used to report compliance with standards. Cohen's Kappa and Intraclass Correlation Coefficient were used for inter- and intrarater reliability based on a $10 \%$ sample of the dataset.

Results Palliative in-patients were significantly more likely than the general population to experience dry mouth $(\mathrm{p}<0.001)$. $86 \%$ of admissions had OHAT completed and 91\% of these were on day of admission. Care plans were completed for $76 \%$ of patients with oral care needs. Appropriate medications were prescribed for $34 \%$ of patients with dry mouth. Interand intra-rater reliabilities were high or perfect for all primary outcomes.

Conclusions Results indicate that oral health is evaluated in the majority of patients, however treatment appears low. This may be partly due to poor instrument design, where non-prescription treatments or 'treatment unnecessary' cannot be documented. Existing tools could be amended to reflect patient care needs more accurately. A change project is currently underway within the care setting to improve practice as a result of the study.

\section{P-25 AUDIT OF OPIOID PRESCRIBING IN A HOSPICE IN- PATIENT UNIT}

Nicola Towner, Helen McGee. Hospice in the Weald, Pembury, UK

10.1136/bmjspcare-2017-00133.25

Background Morphine is the recommended first line opioid by NICE for severe pain. However, many patients on the Hospice in the Weald ward were noted to be on alternative opioids such as Oxycodone and Fentanyl. The reason for choosing an alternative opioid was not always clear. It is accepted practice that oxycodone is a suitable alternative for those who develop adverse effects with morphine that are not controlled by usual measures and Fentanyl and Alfentanil are suitable with poor renal function.

Aims This audit aimed to identify if prescribing of opioids in IPU followed both NICE and local guidelines.
Methods This prospective audit ran over a 6 week period during November and December 2015. Once a patient had been identified as being prescribed an alternative opioid information was obtained by talking to the patient or relatives if appropriate and/or looking through paper notes, electronic entries, telephoning GP surgeries or community pharmacies.

Results 38 patients were admitted to IPU during the audit period of whom $47 \%$ were prescribed an alternative opioid. The most common reasons for switching a patient to an alternative opioid were due to renal function (33\%) or patient being intolerant of morphine (39\%). The most commonly reported side effect leading to a change in opioid was hallucinations and in $71 \%$ of all cases a decreased dose of medication was tried before switching opioids. $72 \%$ of switches to an alternative opioid were deemed appropriate according to NICE and local guidelines.

Conclusions Although alternative opioids are frequently prescribed on IPU, the majority of prescribing is appropriate according to NICE and local guidelines. Improvements could be made in documenting in the electronic notes the reason an alternative opioid was prescribed, side effects experienced and whether a reduced dose or supportive medication was tried first.

\section{P-26 CHALLENGING THE PRESSURE ON NHS RESOURCES: COULD 48-HOUR CONTINUOUS SUBCUTANEOUS INFUSIONS (CSCIS) HELP? A SYSTEMATICALLY- STRUCTURED REVIEW OF THE CURRENT EVIDENCE BASE}

\begin{abstract}
1,2 James Baker, ${ }^{2,3}$ Andrew Dickman, ${ }^{2}$ Stephen Mason, ${ }^{1,2}$ John Ellershaw, ${ }^{1}$ Paul Skipper, ${ }^{4}$ Jenny Schneider. ${ }^{1}$ Royal Liverpool and Broadgreen University Hospitals NHS Trust, Liverpool, UK; ${ }^{2}$ Marie Curie Palliative Care Institute, University of Liverpool, Liverpool, UK: ${ }^{3}$ Blackpool Teaching Hospitals NHS Foundation Trust, Blackpool, UK; ${ }^{4}$ School of Biomedical Sciences and Pharmacy, The University of Newcastle, Newcastle, Australia
\end{abstract}

\subsection{6/bmjspcare-2017-00133.26}

Background The majority of patients express a preference to die at home, yet the most commonly recorded place of death is hospital; in 2012, 36.7\% of deaths in Liverpool occurred in the person's usual place of residence. With an ageing population, NHS resources will be placed under increasing pressure to meet the needs and care preferences of chronically ill patients. Accordingly, innovative approaches to existing therapies are one way to improve care and maximise service delivery. For example, the ability to deliver prescribed medication by CSCI over 48 hours may have numerous benefits in both patient care and health service resource utilisation: current practice limits infusion time to a maximum of 24 hours due to available chemical and microbiological stability data.

Aim To examine the evidence on stability of 48 hour multipledrug syringes/CSCIs in current clinical practice.

Design A systematically-structured review following PRISMA guidelines.

Data sources Three electronic databases (CINAHL, EMBASE and MEDLINE) and grey literature were searched with no time limits. Studies published in English reporting empirical data on the chemical or microbiological stability of continuous subcutaneous infusions or solutions stored in polypropylene syringes were included.

Results Chemical compatibility and stability of 51 different combinations of 12 drugs were reported across the ten studies included in this review. Of the 51 combinations reported, all 